\title{
Art du langage et linguistique du sens
}

Traduction commentée des "Thèses sur le thème "Langage et poésie" " (E. Coseriu)

Language Arts and Linguistics of Meaning. Translation of "Theses about the Topic 'Language and Poetry"'(E. Coseriu)

\section{Christophe Gérard}

\section{(2) OpenEdition}

\section{Journals}

Édition électronique

URL : http://journals.openedition.org/pratiques/4918

DOI : $10.4000 /$ pratiques. 4918

ISSN : 2425-2042

Éditeur

Centre de recherche sur les médiations (CREM)

Référence électronique

Christophe Gérard, «Art du langage et linguistique du sens », Pratiques [En ligne], 179-180 | 2018, mis en ligne le 31 décembre 2018, consulté le 01 mai 2019. URL : http://journals.openedition.org/ pratiques/4918; DOI : 10.4000/pratiques.4918

Ce document a été généré automatiquement le 1 mai 2019.

(C) Tous droits réservés 


\section{Art du langage et linguistique du sens}

Traduction commentée des « Thèses sur le thème "Langage et poésie" " (E. Coseriu)

Language Arts and Linguistics of Meaning. Translation of "Theses about the Topic 'Language and Poetry"'(E. Coseriu)

\section{Christophe Gérard}

Il faut donc considérer le langage poétique comme le langage dans sa pleine fonctionnalité. La poésie - et par poésie je n'entends pas seulement la « poésie » en un sens étroit, mais plutôt la littérature en tant qu'art - est le lieu du déploiement de la plénitude fonctionnelle du langage.

E. Coseriu (2007a, p. 148)

1 Quelle relation la poésie entretient-elle avec le langage? Est-elle un usage linguistique parmi d'autres qui, comme par exemple le parler scientifique ou l'expression quotidienne, limite le potentiel du langage - conçu comme pure «activité libre ou créatrice » (Coseriu, 2001, p. 433) ? Ou bien la nature même de la poésie ne conduit-elle pas, bien plutôt, à ne pas la différencier du langage ainsi défini, en concevant qu'il existe une identité fondamentale entre le langage et la poésie, comme le défendait B. Croce (1951) ? C'est ce problème fondamental qui est au centre des «Thèses sur le thème "langage et poésie" $»^{1}$, dont nous produisons ici la traduction inédite ${ }^{2}$.

2 Fondamental, ce problème l'est en effet à plus d'un titre. D'abord, parce que le problème de l'identité du langage et de la poésie occupe toute l'histoire de la «stylistique moderne romane $»^{3}$. Ensuite, pour ces trois autres raisons, que nous développerons dans les pages suivantes :

3 a) concernant les différentes manières de concevoir la littérature, affirmer une telle identité revient à se positionner contre la théorie de l'écart et ses différentes guises ${ }^{4}$, non 
seulement en donnant une définition de la poésie comprise comme activité universelle, mais aussi en portant la réflexion sur le plan social et historique, pour préciser comment, en tant qu'art du langage, la poésie se réalise nécessairement dans des traditions discursives, littéraires ou non ;

b) concernant les fondements de la théorie linguistique, répondre à ce problème n'implique pas seulement les notions de poésie et de langage, et leur conceptualisation : y répondre engage le linguiste à formuler une conception du sens - soit, dans le cas présent, la sémiotique textuelle et la méthode d'interprétation des œuvres littéraires qu'E. Coseriu relie au sein d'une linguistique $d u$ sens, contexte hors duquel on ne peut complétement comprendre les TLP ;

c) concernant l'étude linguistique de la littérature et sa méthodologie, concevoir l'identité du langage et de la poésie, d'une part, et se doter de la linguistique du sens qui lui correspond, d'autre part, permet de s'opposer à diverses postures réductionnistes en visant une caractérisation linguistique des œuvres littéraires qui fasse droit à leur complexité, au moyen d'un éclectisme raisonné.

\section{Poésie, langage et traditions discursives ${ }^{5}$}

E. Coseriu hérite le thème de l'identification de la poésie et du langage de l'esthétique linguistique de B. Croce, qui le recevait à son tour de G. Vico (Scienza nuova, 1725) :

Langage et poésie sont pour Vico substantiellement identiques. Réfutant cette " commune erreur des grammairiens, qui disent que le parler de la prose est né avant, et celui du vers après ", il trouve "dans les origines de la poésie telles qu'on les a ici découvertes », les « origines des langues, et l'origine des lettres ». [...] Il put réfuter l'autre commune erreur des grammairiens: «que le parler des prosateurs est propre, et impropre celui des poètes ». Les tropes poétiques, qui se rangent sous l'espèce des métonymies, lui apparurent «nés de la nature des premières nations, non du caprice d'hommes particuliers, habiles en poésie » [...]. (Croce, 1904).

G. Vico voyait alors dans la poésie un principe anthropologique, et ses conceptions, pour l'historien de l'esthétique que fut $\mathrm{B}$. Croce, restèrent en la matière longtemps inégalées (cf. e.g. l'analogie du langage et de l'art chez W. von Humboldt). De fait, le thème traité dans les TLP déborde la linguistique et, par exemple, chez E. Coseriu, la compréhension des références à $\mathrm{B}$. Croce passe à l'évidence par la pensée esthétique, c'est-à-dire en particulier par la théorie crocéene de l'art, et sans doute aussi par la réflexion esthétique menée par E. Coseriu lui-même ${ }^{6}$. Dans ce contexte, la «poésie» doit être globalement comprise comme un mode de l'activité artistique humaine (parmi d'autres: peinture, musique, architecture, etc.), et en soi comme l'art du langage verbal par excellence.

Dans le domaine du langage verbal, la particularité des TLP est de soutenir une affirmation qui contredit la doxa: il ne faut pas se représenter la poésie comme un " écart » par rapport au langage quotidien, car c'est le langage quotidien qui réalise bien plutôt un écart par rapport au langage poétique. De fait, la poésie est le mode par lequel le langage s'exprime ou s'accomplit dans sa plénitude, sans restrictions fonctionnelles $a$ priori, à la différence par exemple de ce qu'on observe dans le langage quotidien, scientifique, religieux, etc. C'est précisément cette absence de restrictions fonctionnelles qui, pour E. Coseriu, est au fondement d'une identification de la poésie au langage :

Il faut donc considérer le langage poétique comme le langage dans sa pleine

fonctionnalité. La poésie - et par poésie je n'entends pas seulement la «poésie » en 
un sens étroit, mais plutôt la littérature en tant qu'art - est le lieu du déploiement

de la plénitude fonctionnelle du langage (Coseriu, 2007a, p. 148). de type littéraire, où sa manifestation n'est ni limitée à un « registre » particulier (poésie lyrique, mystique, engagée, scientifique, etc.), ni à un genre poétique particulier (sonnet, ballade, poème en prose, haiku, etc.) ni à aucune forme particulière (poésie versifiée, vers libre, calligramme, ponctuation «blanche » [Favriaud, 2004a; Favriaud, 2014b, p. 45-64], etc.).

Mais si les traditions discursives littéraires sont intimement liées à la poésie, par nature pour ainsi dire, la poésie ne leur est pourtant pas assujettie, car celle-ci est en soi, comme le langage, pure "activité libre ou créatrice » (Coseriu, 2001, p. 433). De fait, dans la mesure où la poésie ne se voit appliquer aucune restriction fonctionnelle, elle doit même pouvoir s'exprimer sans restriction de domaine d'usage, c'est-à-dire pouvoir se manifester par-delà ce qu'on appelle « littérature ». En effet,

Un observateur attentif des formes quotidiennes de l'expression peut aussi voir comment, dans un flux vivant, les mots y sont perpétuellement renouvelés de manière imaginative et comment éclot une poésie variée, sérieuse et sublime, douce, charmante et discrètement souriante ${ }^{8}$.

11 On peut aujourd'hui, par exemple, penser à la tradition urbaine du slam et, plus largement, noter que l'observation de manifestations poétiques, au sens étymologique de l'adjectif - c'est-à-dire d'une créativité langagière - dans les communications les plus quotidiennes, ne permet pas d'attribuer à la poésie un rapport exclusif à la littérature.

Ainsi, la poésie, le langage poétique, renvoie certes à une situation discursive privilégiée (la littérature et ses différentes traditions discursives) mais aussi, au-delà, à un mode d'existence observable dans des traditions discursives non-littéraires, notamment sous la forme des innombrables néologismes produits dans la presse, dans la parole enfantine, sur les réseaux sociaux, etc.

\section{Linguistique et littérature : la méthode en question}

\section{La littérature au péril des réductionnismes : donner le primat au sens}

13 Les querelles de spécialistes qui, dans la deuxième moitié du $\mathrm{XX}^{\mathrm{e}}$ siècle, marquèrent l'étude de la littérature, et en particulier celle de la poésie' ${ }^{9}$ ont été attisées par des prises de position dogmatiques et diverses conceptions réductionnistes. Pour tirer les leçons de ce passé, le linguiste devrait notamment, aujourd'hui, s'interdire des étroitesses de vue que dénonçait déjà H. Meschonnic (1969, p. 15) :

L'étude du langage ne peut pas ne pas interroger la littérature, qui est langage, et communication. Et si elle est langage, une première illusion serait de poser un privilège exclusif de la linguistique sur la littérature. Jusqu'à l'illusion des modèles qui épuiseraient l'œuvre. Tout ne se réduit pas à du linguistique. Le texte est un rapport au monde et à l'histoire. Une illusion inverse serait de prendre la linguistique pour une auxiliaire, qui procurerait un matériau à élaborer ensuite, une étape en somme avant de parvenir aux constituants fondamentaux de la littérature (la connaissance psychologique, sociologique...), et c'est le dualisme des « littéraires ». 
14

Au-delà, il doit être clair que le péril réductionniste guette toute approche linguistique des textes littéraires qui s'affranchit d'une conception adéquate du texte, du style, du genre textuel ${ }^{10}$ et, couronnant tout cela, d'une conception du sens appropriée à l'objet culturel qu'est toute œuvre d'art. définisse ces deux notions : de la création du texte à sa réception, la poésie ne cesse jamais d'être langage, expression d'une intention signifiante et support d'actes interprétatifs et, par conséquent, sens. Aussi, seul le sens semble être affranchi de la variabilité historique des «registres», des genres et des formes (supra 1). D'autre part, dès lors qu'elle ne conceptualise pas le sens, entendu comme le produit de l'interprétation d'une œuvre et de ses passages, toute réflexion ou analyse linguistique d'un objet poétique (texte particulier, tradition poétique ou poésie en général) voit sa portée nécessairement réduite ${ }^{11}$.

Étudier la poésie comme art du langage nécessite donc de donner le primat à la problématique du sens et de l'interprétation, tant d'un point de vue théorique que méthodologique (Rastier, 2001a). À cet égard, les propositions de G. Dessons et H. Meschonnic (1998) sur la «signifiance», de F. Rastier (2006) sur les «formes sémantiques ", de M. Dominicy (2011) sur l'« évocation", d'I. Fónagy (1983) sur l'expressivité phonétique, etc., méritent une égale attention. Toutefois, l'opposition au réductionnisme apparait plus radicale dans la sémiotique d'E. Coseriu (infra Le sens selon E. Coseriu) ${ }^{12}$, son projet intellectuel ayant précisément consisté à élaborer une «linguistique intégrale ", propre à ne privilégier ni à négliger aucun des multiples aspects du langage $\mathrm{e}^{13}$.

\section{Pour un éclectisme raisonné}

17 Au-delà du réductionnisme, l'étude des textes littéraires rencontre l'éclectisme, qui caractérise aujourd'hui les rapports méthodologiques entre linguistique et poésie, comme le notent J. Gardes Tamine et M. Monte (2007, s.p.) dans leur introduction au numéro de Semen « Linguistique et poésie : état des lieux et perspectives » :

D'une certaine façon, c'est l'éclectisme, ou le choix d'un seul niveau d'analyse, qui domine le plus souvent dans ces différentes contributions: Lucien Victor s'interroge sur le rôle de la syntaxe en poésie, et sur son évolution possible au cours des siècles, Hugues Laroche montre les changements dans le lexique poétique au XIX ${ }^{e}$ siècle, Jean-Michel Gouvard analyse la métrique d'un recueil de Bonnefoy.

D'un côté, d'un point de vue anti-dogmatique, l'éclectisme apparait comme une pratique tout à fait légitime: une pluralité d'approches distinctes étant reconnues comme également valides, il devient possible de les combiner pour éclairer, au moyen de leurs apports particuliers, la compréhension des œuvres et de la littérature en général. Mais la pratique de l'éclectisme, qui épistémologiquement témoigne d'une absence d'unité disciplinaire ${ }^{14}$, revient en fait à user d'une stratégie d'analyse subjective et individuelle, généralement dénuée de principes explicites, dont l'efficacité dépend de l'expérience et de l'ingéniosité de celui ou celle qui l'élabore. En ce sens, l'éclectisme est moins une méthode qu'une pratique d'analyse ad hoc.

Actuellement, cependant, l'étude des œuvres littéraires apparait irrémédiablement liée à l'éclectisme : 1) la linguistique est devenue un champ composite en partie fait de théories divergentes et d'un métalangage polysémique ${ }^{15} ; 2$ ) les disciplines du texte (rhétorique,

Pratiques, 179-180 | 2018 
stylistique, poétique, thématique, etc.) constituent un champ foncièrement hétérogène ${ }^{16}$; 3) la complexité structurelle et interprétative des textes littéraires parait défier toute invention d'une méthode unifiée, cette complexité se modifiant de surcroit au gré de changements historiques permanents (succession des courants littéraires, émergence de nouveaux genres et évolution de ces derniers, multiplication des styles individuels, etc.); 4) enfin, remplacer l'éclectisme par l'application d'une conception/méthode particulière, jouissant d'une relative autonomie, ne résout pas le problème ${ }^{17}$.

Si l'éclectisme ne peut être dépassé, au sens strict du terme, il pourrait néanmoins être rationnalisé en se fondant sur une conception du langage et des œuvres littéraires suffisamment générale et systématisée pour permettre de fédérer une diversité d'approches particulières. La linguistique du sens d'E. Coseriu, que condensent les TLP, contribue clairement à cette réflexion - dont l'objectif ultime est de passer d'une pratique individuelle à une méthode générale - en articulant une sémiotique textuelle et une herméneutique philologique.

\section{Sémiotique textuelle et herméneutique philologique}

\section{Le sens selon E. Coseriu}

21 La « linguistique intégrale » d'E. Coseriu répondait, par anticipation (dès les années 1950), à la parcellisation actuelle des sciences du langage ${ }^{18}$. De manière analogue, sa linguistique du texte (Coseriu 2007a) repose sur un modèle sémiotique qui a vocation à concevoir tous les aspects entrant, nécessairement ou potentiellement, dans la constitution du sens, sans privilégier l'étude d'aucun en particulier.

Comment le sens est-il défini au sein de ce modèle? Dans les TLP, la problématique du sens est omniprésente, dès les trois premiers paragraphes (I.1, I.2 et I.3) et le plus explicitement dans les deux derniers (IV.2 et IV.3); toutefois aucune définition du sens n'est formulée. Pour en trouver une clairement exposée, il faut lire d'autres textes d'E. Coseriu, notamment l'ouvrage Textlinguistik: "L'émergence du sens résulte de la combinaison des fonctions de Bühler (fonctions de représentation, d'expression et d'appel) et de l'évocation » (Coseriu, 2007a, p. 137, trad. CG).

Contrairement au modèle du signe de K. Bühler $(1972,1982)$, bien connu, le terme d' évocation, forgé par E. Coseriu, demande ici une explication, d'autant que ce mot d'usage courant peut, on le sait, prendre un contenu tout différent au sein d'autres théories (Dominicy, 2011).

Si, chez E. Coseriu, l'évocation est inhérente à la conceptualisation du sens, ce concept sert en premier lieu à préciser le fonctionnement du signe verbal en contexte. En effet, l' évocation désigne l'ensemble des relations qu'un signe, outre les fonctions de K. Bühler, peut entretenir avec d'autres signes " en langue ", avec d'autres signes au sein d'un texte, mais aussi avec des "choses", avec la «connaissance du monde» et avec différents entours $^{19}$. Rapidement énumérées dans le paragraphe I.2. des TLP, ces relations constitutives de l'évocation sont en fait distribuées au sein d'une classification ${ }^{20}$ dont l'intérêt est d'offrir une représentation systématisée recouvrant un vaste ensemble de phénomènes : rimes, allitérations, champs lexicaux, connotations, formules stéréotypées, onomatopées, synesthésies, phono-symbolisme, mais aussi "univers de discours", sémantisation dynamique du texte, non-dits, etc. De ce fait, le modèle d'E. Coseriu 
correspond à une sémiotique textuelle qui, en visant l'intégralité des relations sémiotiques possibles, répond d'une manière particulièrement adéquate à la poésie, conçue comme « lieu du déploiement de la plénitude fonctionnelle du langage » (TLP, I.8).

Remarquons néanmoins tout d'abord qu'il serait aujourd'hui bien anachronique de s'en tenir au modèle de K. Bühler, car depuis les années 1970 la sémantique lexicale a proposé des modèles plus complexes rendant mieux compte des multiples aspects impliqués dans le fonctionnement du signe linguistique ${ }^{21}$. Ensuite, concernant l'évocation, la classification des relations sémiotiques offre un cadre théorique certes très éclairant mais insuffisant en pratique, car la description des phénomènes visés doit s'appuyer sur un appareil conceptuel plus précis. Par exemple, pour étudier les connotations, qui relèvent de la catégorie des "relations entre un signe et un autre système de signes ", E. Coseriu se fonde sur la théorie de L. Hjelmslev. De même, parmi les "relations du signe à son contexte linguistique ", la constitution dynamique du sens textuel, évoquée dans le passage suivant ne peut être décrite, relativement à un texte particulier, sans faire usage de concepts spécifiques :

Pensez par exemple à un terme qui apparaît dans le titre d'une étude théorique. Il est clair que le "sens" de ce signe, lors de la lecture de cette étude, sera constamment modifié. Au début, on n'a qu'une vague intuition, qu'une connaissance imprécise de "ce dont il pourrait à peu près être question ». Cette pré-compréhension du signe sera constamment modifiée au cours de la lecture, selon un processus qui, potentiellement, ne s'achève qu'à la fin du texte. (Coseriu, 2007a : 129, trad. CG).

En l'occurrence, pour décrire la constitution dynamique du sens d'un texte, il est possible de s'appuyer sur le concept de «forme sémantique » proposé par F. Rastier (2006).

Ainsi, le modèle d'E. Coseriu, bien loin d'être un modèle à part parmi d'autres conceptions sémantiques (G. Dessons/H. Meschonnic, F. Rastier, M. Dominicy, I. Fónagy, etc.), appelle bien plutôt ces dernières comme autant d'adjuvants théoriques pouvant répondre, au moyen de leurs spécificités conceptuelles, à un type de phénomène sémiotique reconnu par un même modèle de référence. Mieux, la généralité même du modèle cosérien offre une vision d'ensemble qui permet de dépasser une représentation par trop isolationniste de conceptions ou théories partielles, tout en étant conscient de la cohérence globale qui les lie. De cette façon, face au texte littéraire notamment, le linguiste peut pratiquer un éclectisme raisonné, à défaut de disposer aujourd'hui d'une méthode unifiée.

\section{Interpréter le sens des textes}

Mais si la linguistique du sens d'E. Coseriu permet d'aborder les œuvres littéraires sans en réduire la complexité c'est aussi parce que son modèle sémiotique prend toute sa valeur dans une réflexion herméneutique, qui se traduit concrètement par la proposition d'une méthode d'interprétation.

En effet, il ne suffit pas de définir le sens d'un point de vue analytique comme «la combinaison des fonctions de Bühler (fonctions de représentation, d'expression et d'appel) et de l'évocation ». Car au-delà d'une combinaison, le sens se comprend comme l'objet d'une intention qui cherche à comprendre «ce que signifie» un texte. Par exemple, dans la célèbre nouvelle de F. Kafka, on peut se demander ce que signifie, en définitive, la métamorphose de Grégor Samsa en insecte monstrueux. 
30 Dans ce cas, de la perspective d'E. Coseriu, on cherche à savoir ce que cette métamorphose " veut dire » en s'appuyant sur la signification et la désignation des signes

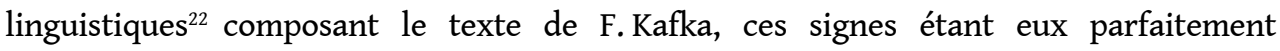
identifiables et compréhensibles. Plus exactement, comme l'exposent les TLP (IV.2, IV.3), dans l'acte d'interprétation, la signification et la désignation sont conçues comme un plan sémiotique qu'il convient de dépasser pour constituer le signe textuel dans sa globalité : « la signification et la désignation constituent ensemble le signifiant, le sens constituant en revanche le signifié du signe textuel » (Coseriu 2007a, p. 64-65, trad. CG). Autrement dit, interpréter un texte consiste à passer par un premier niveau sémiotique, celui de la signification et de la désignation, pour constituer un sens à un second niveau sémiotique, considéré comme supérieur, en actualisant une partie des nombreuses relations de l' évocation (TLP I.2) :

Il est théoriquement possible qu'on comprenne tout ce qui est dit au premier niveau sémiotique [signification et désignation, $C G$ ] sans saisir la moindre chose au second niveau sémiotique. Autrement dit: il est possible de raconter en détail la métamorphose de Kafka, qu'on peut même connaître par cœur, sans qu'on soit capable d'en dire quelque chose, sur ce que cette métamorphose a elle-même à « dire ", sur son « sens » (ibid., p. 66, trad. CG).

31 E. Coseriu relit ainsi Don Quichotte en argumentant que le roman de M. de Cervantès exprime les limites du combat pour la liberté, notamment (ibid., p. 167-171). Cette lecture permet du reste de voir que, pour E. Coseriu, l'établissement « du sens » d'un texte peut parfaitement admettre une pluralité de sens compossibles.

En tant que «signifié du signe textuel ", un tel sens ne correspond ni, par exemple, à l'évocation de M. Dominicy (2011), à laquelle l'interprète recourt naturellement pour réaliser sa tâche, ni chez F. Rastier $(2001 \mathrm{a} ; 2006)$ à la constitution interprétative de fonds et de formes sémantiques (contenus thématiques, narratifs, énonciatifs, etc.), qui se situent exclusivement au niveau de la textualité.

Pour établir cette sorte de sens, E. Coseriu (2007a, p. 153-155, trad. CG) propose une méthode d'interprétation qui est une transposition, adaptée à la grande complexité du texte, de la méthode des commutations :

Chaque analyse linguistique part, implicitement ou explicitement, d'une fonction préétablie, pour constater dans un second temps comment cette fonction se manifeste dans la langue concernée et comment les différentes fonctions se comportent entre elles. [...]. On remplace des parties de l'expression d'un signe pour constater si le contenu s'en trouve changé ou non. On utilise, consciemment ou inconsciemment, la méthode des commutations. [...]. En principe, cette méthode peut être appliquée d'une façon tout aussi consciente et systématique que la technique des paires minimales dans le domaine de la phonologie.

Cette méthode et ses applications à différents textes poétiques (de Sappho, d'Eschyle, etc.) méritent d'être discutées à la lumière de l'histoire de l'herméneutique ${ }^{23}$. Elles sont néanmoins caractéristiques d'une linguistique du texte comprise comme linguistique du sens, dont la tâche consiste précisément en la description et l'établissement de cette sorte de sens. Selon nous, pour paraphraser la dernière phrase des TLP, l'étude linguistique des œuvres littéraires doit avoir cette ambition si elle veut être adéquate à son objet. 


\section{Eugenio Coseriu}

\section{Thèses sur le thème " Langage et poésie " 24} souvent réduites ou "désactualisées» (rendues inopérantes) dans les différentes modalités de l'usage linguistique (par exemple, dans le langage de la vie quotidienne, dans le langage scientifique, etc.). qui leur sont associées, peut être constatée dans ce qu'on appelle le « langage poétique ». 

précisément la suppression d'une négativité, la levée d'une restriction (l'« automatisation ») et par là même la restitution, c'est-à-dire le rétablissement de la pleine fonctionnalité du langage en tant que tel. D'une manière analogue, on peut interpréter la fonction poétique de Jakobson comme une fonction qui concerne le «message » lui-même, c'est-à-dire comme un « usage linguistique » dans lequel le dit (ce qui est dit) vaut simplement comme dit: ce qui ne signifie pas autre chose que la parole poétique est un « dire absolu ».

6. C'est pourquoi le langage poétique ne peut être compris comme un usage linguistique parmi d'autres, mais plutôt comme le langage par excellence, c'est-à-dire comme réalisation de toutes les possibilités du langage en tant que tel.

7. Il s'ensuit que le langage poétique ne peut pas être conçu comme la réduction du langage à une fonction particulière, qu'on a coutume d'appeler "fonction poétique », pas plus qu'il ne peut être compris comme adjonction de cette fonction poétique au langage (langage + fonction poétique). D'un côté, le langage poétique ne peut nullement être considéré comme une réduction du langage. De l'autre, aucune fonction particulière ne s'ajoute, car les différentes possibilités qui sont actualisées dans le langage poétique appartiennent déjà au langage en tant que tel.

8. Il faut conclure de ces réflexions que le langage poétique représente la pleine fonctionnalité du langage, autrement dit que la poésie (la « littérature » comme art) est le lieu du déploiement de la plénitude fonctionnelle du langage.

9. La poésie n'est pas, comme on le pense, un «écart " (déviance) ${ }^{33}$ par rapport à un langage « ordinaire » (qu'on se représente comme le langage «normal »), mais c'est bien plutôt le langage de la vie quotidienne qui accomplit un écart par rapport à un langage que l'on pourrait dire total ou intégral. Ceci vaut aussi pour les autres modalités de l'usage linguistique (par exemple, pour le langage scientifique) : en effet, chacune de ces modalités n'advient qu'en raison d'une forte réduction du langage en tant que tel, lequel coïncide avec le langage de la poésie.

10. C'est du reste le sens exact de la définition du langage poétique comme «langage désautomatisé ", tel que le concevait l'École de Prague $^{34}$. Ici, le privatif dé- signifie 11. En découle une série de conséquences pour la linguistique du texte, qui ne valent pas seulement pour l'étude linguistique des textes littéraires. Entre autres, les suivantes :

a) On peut certes développer une théorie des possibilités des textes, mais pas une méthode générale de l'interprétation des textes entendue comme discovery procedure ${ }^{35}$, car il est impossible de prédire quelles relations entre les signes se présenteront comme actualisées dans un texte particulier. En effet, ces relations sémiologiques doivent être établies pour chaque texte, c'est-à-dire "découvertes " $^{36}$. Il en va ainsi des rapports entre la linguistique générale des textes et l'interprétation d'un texte comme des rapports entre la grammaire générale, qui concerne les possibilités fonctionnelles du langage, et la grammaire d'une langue particulière, au sein de laquelle il ne peut s'agir que de constater la réalisation historique de possibilités fonctionnelles générales.

b) Tous les « effets » d'un texte trouvent leur origine dans le texte lui-même (le contexte compris) au moyen des relations qui s'y actualisent. Ces effets sont tous déterminés par le texte lui-même et peuvent ainsi se voir objectivement constatés, justifiés et analysés. Du reste, sur la dimension matérielle rien n'est " prévisible ", pas même l'unité matérielle du texte, car l'unité proprement dite (l'unité de «sens») peut justement être donnée en l'absence de sa matérialité. 
51 c) Il faut considérer les textes littéraires comme des modèles optimaux pour la linguistique du texte parce que, précisément, cette sorte de textes présente la plus grande richesse fonctionnelle, et par ailleurs parce que pour toutes les autres sortes de textes on constate d'inévitables "automatisations" (qui sont, comme on l'a dit, autant de « désactualisations » fonctionnelles du langage).

52 《stylistique de l'écart »37, c'est-à-dire la stylistique qui caractérise la langue d'un poète (ou d'un écrivain) comme déviance, comme « usage particulier » ou " originalité » par rapport à ce qu'on appelle habituellement la «langue courante ", n'atteint qu'un résultat stérile dans le cas précis des grands poètes (ou écrivains). Par exemple, il est impossible de caractériser la langue de Dante comme un usage spécial existant à l'intérieur de l'italien. La langue des grands poètes semble simplement coïncider avec la langue historique - comme réalisation des possibilités déjà données en elle. Par là, une langue historique est à certains égards identique à la langue poétique correspondante, et à cet égard il n'y a vraiment rien d'absurde à parler de l'italien comme de la «langue de Dante » ou de l'anglais comme de la « langue de Shakespeare ». langues historiques. En effet, loin d'être des "classes» (et par là, loin d'être des "genres" au sens propre du terme), ce sont bien plutôt des individus historiques, exactement comme les langues. En toute rigueur, il est impossible de définir le roman ou la tragédie en tant que classes. On peut seulement décrire le roman en tant que genre historiquement transmis ou la tragédie en tant qu'elle est historiquement transmise et en étudier l'évolution historique. Et il en va de même pour les langues historiques. Aussi, par exemple, est-il impossible de définir l'allemand : en tant qu'individu historique, la langue allemande ne peut qu'être décrite synchroniquement et étudiée historiquement. Ce parallèle entre les genres littéraires et les langues semble lui aussi indiquer la même identité entre le langage et la poésie.

En effet, comme unité de l'intuition et de l'expression ${ }^{38}$, c'est-à-dire en tant que pure création de significations (qui correspondent à l'«être des choses»)" et si nous considérons le sujet créateur comme absolu (c'est-à-dire uniquement dans sa relation à ce qu'il crée), le langage peut effectivement être assimilé à la poésie, car la poésie correspond, précisément, à la saisie intuitive de l'être. En effet, tout comme le langage, la poésie ne suppose aucune distinction préalable entre le vrai et le faux, entre existence et inexistence. Aussi bien le langage que la poésie demeurent antérieurs à ces distinctions. D'un autre côté, comme le langage, la poésie est une saisie de l'universel dans l'individuel, une objectivation des contenus intuitifs de la conscience. Le langage absolu est ainsi poésie. C'est ce que laissent entendre ou ce que défendent différents philosophes, notamment Croce qui a minutieusement donné ses raisons pour fonder cette identité.

1. Malgré tout, l'identification du langage à la poésie n'est pas recevable parce que, précisément, le langage n'est pas absolu. En effet, l'objectivation de l'intuition, le rapport entre le créateur de langage et ce qu'il crée, n'est qu'une dimension du langage. Mais le langage proprement dit possède une autre dimension, la dimension donnée par l'« altérité » du sujet, par le fait que la conscience créatrice de langage est une conscience ouverte vers d'autres consciences ${ }^{40}$. Ce principe ne se laisse pas simplement interpréter 
comme échange d'information, c'est-à-dire comme communication de quelque chose $e^{41}$. É tant d'ordre pratique, la communication comme échange d'information peut en effet ne pas s'établir dans certaines circonstances, et doit donc être distinguée de la communication avec un autre, qui est elle, en revanche, présupposée pour tout acte linguistique. Par conséquent, comme création linguistique primaire, le langage s'adresse toujours nécessairement à quelqu'un d'autre, à la différence de la poésie, qui est langage absolu.

2. Comme activité d'un sujet toujours "relatif» (doté d'«altérité»), le langage est appréhension et structuration du monde, sans cependant être interprétation du monde ou création de mondes possibles. En revanche, la poésie est toujours absolue et, précisément, elle crée aussi d'autres mondes possibles. Par conséquent, il convient de concevoir la poésie comme une "absolutisation" du langage, une absolutisation qui, cependant, n'advient pas au plan du langage en tant que tel, mais bien plutôt au plan du sens du texte. En poésie, tout ce qui est signifié et désigné au moyen du langage (attitudes, personnages, situations, événements, actions, etc.) devient un signifiant dont le signifié n'est autre, précisément, que le sens du texte ${ }^{42}$. Dans cette perspective, par exemple, Kafka ne parle à vrai dire pas de Gregor Samsa, mais au moyen de Gregor Samsa à propos d'autre chose ; de ce point de vue, Gregor Samsa n'est lui aussi qu'un simple signifiant.

3. Le langage en tant que tel est certes porteur de signification, mais jamais de « sens » : il rend seulement possibles toutes sortes de sens qui, cependant, n'apparaissent que dans les textes. Par conséquent, les textes ne peuvent pas simplement être conçus comme des manifestations du langage en tant que tel, mais plutôt comme une modalité supérieure du linguistique, en laquelle le langage en tant que tel devient expression pour des contenus situés à un niveau sémiotique supérieur, celui du sens ${ }^{43}$. La linguistique du texte doit tenir compte de ce fait si elle veut être adéquate à son objet.

\section{BIBLIOGRAPHIE}

ADAM, J.-M. (2005). « Qu'avons-nous fait des espoirs mis dans l'analyse de discours et la théorie du texte? ». In : Jacquet-Pfau, C., Sablayrolles, J.-F. \& Pruvost, J. (éds), Mais que font les linguistes ? Les sciences du langage, vingt ans après. Paris : L'Harmattan, p. 109-120.

ADAM, J.-M. (2010). «L'émergence de la Linguistique Textuelle en France : entre perspective fonctionnelle de la phrase, grammaires et linguistiques du texte et du discours ", Revista Investigações 23, p. 11-47.

ASCHENBERG, H. (2015). «Subjectivité et objectivité dans la pensée de Coseriu ». In : Gérard, C. \&

Missire, R. (éds), Eugenio Coseriu aujourd'hui. Linguistique et philosophie du langage. Limoges :

Lambert-Lucas, p. 207-219.

BLANCHE-BENVENISTE, C. (2005). «L'étude de la morphologie et de la syntaxe de la langue ». In : Jacquet-Pfau, C., Sablayrolles, J.-F. \& Pruvost, J. (éds), Mais que font les linguistes ? Les sciences du langage, vingt ans après. Paris : L'Harmattan, p. 73-92.

BLANK, A. (1997). Prinzipien des lexikalischen Bedeutungswandels am Beispiel der romanischen Sprachen. Tübingen : Niemeyer. 
BLANK, A. (2001). Einführung in die lexikalische Semantik für Romanisten. Tübingen : Niemeyer.

BÜHLER, K. (1972) [1933]. « Die Axiomatik der Sprachwissenschaften ». Kant-Studien 38, p. 19-90.

BÜHLER, K. (1982) [1934]. Sprachtheorie. Die Darstellungsfunktion der Sprache. Stuttgart : G. Fischer.

CABRÉ CASTELLVÍ, M. T. (2006). « La clasificación de neologismos: una tarea compleja ». Alfa 50 (2),

p. 229-250.

COSERIU, E. (1971). « Thesen zum Thema “Sprache und Dichtung” ». In : Stempel, W.-D. (éd.), Beiträge zur Textlinguistik. Munich : W. Fink, p. 183-188.

COSERIU, E. (2001). L'homme et son langage. Louvain/Paris/Sterling : Peeters.

COSERIU, E. (2003). Geschichte der Sprachphilosophie. Tübingen : A. Francke.

COSERIU, E. (2007a) [1980]. Textlinguistik. Eine Einführung. Tübingen : Narr.

COSERIU, E. (2007b) [1973]. « Synchronie, diachronie et histoire. Le problème du changement linguistique ». Texto! 12 (3-4). Trad. par T. Verjans. En ligne : http://www.revuetexto.net/1996-2007/Parutions/Parutions.html.

CROCE, B. (1904). Esthétique comme science de l'expression et linguistique générale. Trad. de l'italien par H. Bigot. Paris : V. Giard et E. Brière.

CROCE, B. (1951). La poésie. Introduction à la critique et à l'histoire de la poésie et de la littérature. Paris : Presses universitaires de France.

De mauro, T. (1994). «Antonio Pagliaro and Semantic Criticism ». In : De Mauro, T. \& Formigari, L. (éds), Italian Studies in Linguistic Historiography. Münster : Nodus, p. 39-44.

DELCROIX, M. \& GEERTS, W. (éds) (1980). «Les Chats » de Baudelaire. Une confrontation de méthodes. Namur/Paris : Presses universitaires de Namur/Presses universitaires de France.

DEssons, G. \& MESCHONNIC, H. (1998). Traité du rythme. Des vers et des proses. Paris : Dunod.

Dominicy, M. (2011). Poétique de l'évocation. Paris : Classiques Garnier.

EBELING, G. (1959). « Hermeneutik ». In : Galling, K. (éd.), Die Religion in Geschichte und Gegenwart. Tübingen : J. C. B Mohr/P. Siebeck, p. 242-262.

FAVRIAUD, M. (2004a). «Quelques éléments d'une théorie de la ponctuation blanche - par la poésie contemporaine ». L'information grammaticale 102, p. 18-23. En ligne : https://www.persee.fr/doc/ igram_0222-9838_2004_num_102_1_2559.

FAVRIAUD, M. (2014b). Le plurisystème ponctuationnel français à l'épreuve de la poésie contemporaine. Limoges : Lambert-Lucas.

FóNAGY, I. (1983). La vive voix, essais de psycho-phonétique. Paris : Payot.

GARDES TAMINE, J. \& MONTE, M. (2007). «Introduction ». Semen 24. En ligne : http:// journals.openedition.org/semen/6583.

GÉRARD, C. (2005a). Contribution à une sémantique interprétative des styles : Étude de deux cuvres de la modernité poétique. Thèse en linguistique : Université de Toulouse Le Mirail. En ligne : http:// www.revue-texto.net/index.php?id=584 [Texto ! 10 (2)].

GÉRARD, C. (2005b). « Du sens dans la poésie de facture moderniste. L'exemple de "Le grésil” de Jacques Dupin ». Champs du Signe 20, p. 131-154. 
GÉRARD, C. (2009). «Sur l'identité de la poésie et du langage ». Energeia 1, p. 118-127. En ligne : http://www.romling.uni-tuebingen.de/energeia/zeitschrift/2009/sur-l-identite-de-la-poesie-etdu-langage.html.

GÉVAUDAN, P. (2015). « L'énoncé. Notion clé de la théorie du langage ». In : Gérard, C. \& Missire, R. (éds), Eugenio Coseriu aujourd'hui. Linguistique et philosophie du langage. Limoges : Lambert-Lucas, p. 129-146.

GLESSGEN, M.-D. (2012). Linguistique romane. Domaine et méthodes en linguistique française et romane.

Paris : A. Colin.

GRONDIN, J. (2005). « Hermeneutics ». In : Horowitz, M. C. (dir.), New Dictionary of the History of Ideas. New York : C. Scribner's Sons, p. 982-987.

HASSLER, G. (2015). « La relation entre la philosophie du langage et la sémantique chez Coseriu ». In : Gérard, C. \& Missire, R. (éds), Eugenio Coseriu aujourd'hui. Linguistique et philosophie du langage. Limoges : Lambert-Lucas, p. 22-33.

JAKoBSON, R. \& LÉVI-STRAuSs, C. (1962). « “Les Chats” de Charles Baudelaire ». L’Homme 2 (1). p. 5-21.

JENNY, L. (2005). « La langue, le même et l'autre », Fabula-LhT 0. En ligne : http://www.fabula.org/ lht/0/Jenny.html.

KAINZ, F. (1941-1969). Psychologie der Sprache, 5 Bde. Stuttgart : Enke.

косн, Р. (1997). « Diskurstraditionen: zu ihrem sprachtheoretischen Status und ihrer Dynamik». In : Frank, B., Haye, T. \& Tophinke, D. (éds), Gattungen mittelalterlicher Schriftlichkeit. Tübingen : Narr, p. 43-79.

косн, Р. (2015). « Disparition lexicale, variétés linguistiques et traditions discursives ». In : Badiou-Monferran, C. \& Verjans, T. (éds), Disparitions. Contributions à l'étude du changement linguistique. Paris : H. Champion, p. 69-88.

MAIRA, D. (2016). « Roman Jakobson contre Leo Spitzer : militantisme critique et défense d'une méthode ». Romanische Studien 5, p. 135-154. En ligne : http://www.romanischestudien.de/ index.php/rst/article/view/261/829.

MEschonNic, H. (1969). « Pour la poétique ». Langue française 3, p. 14-31. En ligne : https:// www.persee.fr/doc/lfr_0023-8368_1969_num_3_1_5430.

MISSIRE, R. (2018). « Unités linguistiques d'une sémantique discursive », Langages 210 (2), p. 17-34. PÊCHEUX, M. (1990). L’inquiétude du discours. Paris : Éd. des Cendres.

PRUVOST, J. \& SABLAYROLLES, J.-F. (2016) [2003]. Les néologismes. Paris : Presses universitaires de France.

RAIBLE, W. (1983). « Zur Einleitung ». In : Stimm, H. \& Raible, W. (dirs), Zur Semantik des Französischen. Wiesbaden : F. Steiner, p. 1-24.

RASTIER, F. (1999). « De la signification au sens. Pour une sémiotique sans ontologie ». Texto! 8 (2-3). En ligne : http://www.revue-texto.net/index.php?id=560.

RASTIER, F. (2001a). Arts et sciences du texte. Paris : Presses universitaires de France.

RASTIER, F. (2001b). « Sémiotique et sciences de la culture ». Linx 44, p. 149-168. En ligne : https:// journals.openedition.org/linx/1058. 
RASTIER, F. (2006). « Formes sémantiques et textualité ». Langages 163, p. 99-114. En ligne : https:// www.cairn.info/revue-langages-2006-3-page-99.htm.

RICHARD, J.-P. (1964). Onze études sur la poésie moderne. Paris : Éd. Le Seuil.

RIFFATERRE, M. (1971). Essais de stylistique structurale. Paris : Flammarion.

ROUSSET, J. (1962). « Pour une lecture des formes ». In : Rousset, J., Forme et signification. Essais sur les structures littéraires de Corneille à Claudel. Paris : J. Corti, p. i-xxvi.

STAROBINSKI, J. (1970). « Leo Spitzer et la lecture stylistique ». In : Starobinski, J., L'œil vivant II, La relation critique. Paris : Gallimard, p. 34-81.

VILCU, D. (2015). « Le statut épistémologique de la linguistique ». In : Gérard, C. \& Missire, R. (éds), Eugenio Coseriu aujourd'hui. Linguistique et philosophie du langage. Limoges : Lambert-Lucas, p. 51-60.

WACH, J. (1984). Das Verstehen. Grundzüge einer Geschichte der hermeneutischen Theorie im 19.

Jahrhundert. Hildesheim : G. Olms.

\section{NOTES}

1. Désormais abrégé «TLP». Outre ce texte publié en 1971, deux autres écrits d'E. Coseriu traitent du même sujet. Ils portent le titre «Langage et poésie » [Sprache und Dichtung] et présentent des développements plus approfondis sur certains aspects : le chapitre manuscrit du cours de 1963-1964 (infra n.3), publié dans C.Gérard (2009), et la section de chapitre de Textlinguistik. Eine Einführung (Coseriu 2007a, p. 146-149) ouvrage publié en 1980 par son élève J. Albrecht, à partir de notes de cours.

2. Traduction d'E. Coseriu (1971). La version française que nous livrons vient s'ajouter aux versions existant déjà en espagnol et en roumain, dont nous nous sommes en partie inspirés : "Tesis sobre el tema "lenguaje y poesía” » (1979), Lingüística española actual I, 1, p. 181-186. « Teze despre tema "Limbaj şi poezie"»(1995), România literară, XXVIII, 41. Toutes les notes de la version française sont les nôtres.

3. Comme le montra E. Coseriu dans son cours " Moderne romanische Stilforschung ", dispensé entre novembre 1963 et mars 1964, à l'université de Tübingen. Les sources de ce cours inédit sont conservées aux archives Coseriu à Tübingen (http://coseriu.de) sous la forme d'un manuscrit (plus d'une centaine de feuillets in-octavo ainsi qu'une cinquantaine de fiches bristol) et de comptes rendus de séance dactylographiés. La matière du cours est constituée d'une grande diversité de courants (école de Prague, behaviourisme, école de Copenhague, etc.) et d'auteurs (L.Spitzer, T. A. Sebeok, Ch. Bally, B. Croce, M. Fubini, R. Jakobson, L. Hjelmslev, A. A. Hill, V. Chklovski, J. Marouzeau, M. Riffaterre, Devoto, A. Alonso, K. Vossler, Th. Spoerri). Notons qu'E. Coseriu consacre l'avant-dernière partie du cours (quatre séances) et 29 feuillets manuscrits (ni transcrits ni édités) à A. Pagliaro (de Mauro, 1994), dont la «critique sémantique » demeure inconnue en stylistique et linguistique françaises.

4. Sans cesse discutée : e.g. H. Meschonnic (1969), L. Jenny (2005).

5. Nous reprenons ici des passages d'une discussion amorcée dans C. Gérard (2009).

6. Voir le manuscrit « Estética » de 1956, classé en A-XXIII dans les archives Coseriu.

7. Selon le terme forgé par P. Koch (1997, p. 43-45) : «La notion de "tradition discursive" inclut, entre autres, celle de "genre littéraire", mais elle va bien au-delà de celle-ci, passant à travers la grande variété des univers du discours (littérature, histoire, droit, religion, science, etc.). Voici, en vrac, quelques exemples de traditions discursives: style sublime, atticisme, maniérisme; article de fond, sonnet, causerie, blague, chat; actes de langage du baptême, acte de langage du 
serment ; formules de salutation, formule de remerciement, formule de pénalité, etc. » (Koch 2015, p. 79).

8. «Auch bei den alltäglichen Formen des Ausdrucks kann ein aufmerksammer Beobachter sehen, wie entlang des lebendigen Strömens die Wörter fortwährend phantasievoll erneuert werden und erfunden werden und wie eine vieltönige Poesie aufblüht, ernst und erhaben, zart, anmutig und leise lächelnd» (Croce, 1970, Die Dichtung, p. 19).

9. Souvenons-nous de la violente polémique des «Chats» (Delcroix \& Geerts, 1980) suscitée par l'analyse structurale de R. Jakobson et C. Lévi-Strauss (1962).

10. Les exemples sont nombreux (Rastier, 2001a, en particulier les chap. I, VI et VIII). Fondamentalement, toutes ont en commun de négliger le principe d'objectivité propre aux sciences de la culture (Vilcu, 2015).

11. Ce qui est particulièrement net dans le cas de la poésie hermétique, qui exige par nature une prise de position théorique sur le sens (Gérard, 2005a et 2005b).

12. Cette sémiotique particulière doit bien entendu être située et comprise dans une réflexion plus large sur la sémiotique (Rastier, 2001b).

13. Le projet d'une linguistique intégrale - ou intégralisme - entend fédérer une linguistique de la faculté de parler (Linguistik des Sprechens), une linguistique des langues (Linguistik der Sprachen) et une linguistique du texte (Textlinguistik). Il repose sur notamment sur la triade système/norme/ parole, mais aussi energeia/dynamis/ergon et universel/particulier/individuel, ou encore désignation (Bezeichnung)/signification (Bedeutung)/sens (Sinn). Ces triades ont une finalité commune: elles se conjuguent pour former une conception unitaire du langage (pour une présentation en français, Aschenberg, 2015 ; Coseriu, 2001 et, plus partiellement, R. Missire ici même).

14. «Critiques, enseignants, praticiens de l'analyse de contenu usent de notions empruntées à la rhétorique, la stylistique, la sémiotique, la poétique, etc. Cet heureux éclectisme laisse-t-il deviner entre ces disciplines des traditions communes, des emprunts réciproques et peut-être des perspectives complémentaires? Il reste difficile de définir un point de vue général qui pourrait les ordonner, car leur statuts restent disparates. » (Rastier, 2001a, p. 1).

15. " "La" linguistique s'est diversifiée au point que cohabitent des paradigmes différents et des savoirs de plus en plus spécialisés mais, de ce fait, de plus en plus difficilement communicables. Si les linguistes travaillent, beaucoup et souvent bien, leurs recherches et leurs sociolectes se sont babélisés. » (Adam, 2005, p. 110). De fait, « Les linguistes n'ont pas organisé l'entreprise qui consisterait à s'entendre sur un minimum de connaissances admises. [...]. En l'absence de cumul des connaissances, la linguistique française semble actuellement fragile.» (Blanche-Benveniste, 2005, p. 89). Qu'on pense simplement à la polysémie de la notion de « discours » (Pêcheux, 1990 ; Missire, 2018).

16. «Les disciplines du texte [...] diffèrent tant par leur statut épistémologique - et académique que par leurs objectifs, leurs méthodes, leurs procédures de validation; enfin, par le concept même de texte dont elles partent et qu'elles produisent » (Rastier, 2001a, p. 6).

17. Faut-il choisir, en linguistique, entre telle ou telle théorie syntaxique ou morphologique? Entre telle ou telle typologie des procédés néologiques, si importants en poésie (Pruvost \& Sablayrolles, 2016 ; Cabré Castellví, 2006) ? Faut-il choisir, en stylistique, entre la méthode de J. Rousset (1962) et celle de J.-P. Richard (1964) ? Et sur quels critères devrait-on choisir ? Et si les défauts de la méthode de R. Jakobson poussent sans doute à lui préférer les lectures pénétrantes de L. Spitzer (Maira, 2016), ce dernier y parvient moins au moyen d'une méthode au sens strict que par un processus de compréhension de l'œuvre privilégiant l'intuition et la culture du sujetinterprète : «Spitzer était réfractaire à l'idée d'une méthode qui pût être mise entre toutes les mains, et qui fût ainsi devenue un outil universel. Il savait que le terrorisme méthodologique n'est, la plupart du temps, que le cache-misère de l'inculture »(Starobinski, 1970, p. 65). 
18. Alors que F. Rastier (2001b) appelle à «fédérer les sciences de culture " et au « remembrement des sciences du langage » (Rastier, 1999), J.-M. Adam (2010, p. 25) questionne lui l'unification des théories du texte: «les recherches locales sur des langues particulières (travaux sur les connecteurs, les anaphores, les temps verbaux, les cadratifs et autres formes de la modalisation autonymique, la position des adjectifs, les constructions détachées, etc.) peuventelles être intégrées dans un modèle général de la textualité ? Comment mettre ensemble l'abondante production relative au transphrastique, dans des langues différentes de surcroît, et les recherches qui théorisent la textualité générale?»

19. E. Coseriu (2001, p. 54-67) forge le concept d'entour pour rationaliser la notion trop vague de "contexte» et ainsi rendre compte d'une manière systématique de toutes les circonstances conditionnant l'activité des sujets parlants. Sa typologie des entours comprend, à son premier niveau, la situation, la région, le contexte et l'univers de discours (ex. la littérature). Pour une discussion, voir P. Gévaudan (2015) et H. Aschenberg (2015).

20. Dont "Relations avec d'autres signes", "Relations des signes dans d'autres textes", «Relation avec des choses », "Relations avec la connaissance du monde » et « Entours » sont les principales catégories, comprenant elles-mêmes des subdivisions (Coseriu, 2007a, p. 92-136).

21. Voir les synthèses de W. Raible (1983) et de M.-D. Glessgen (2012, p. 273-284) et le modèle d'A. Blank (1997; 2001).

22. Renvoyant au modèle de K. Bühler (voir TLP I.1), la distinction entre désignation et signification est cruciale dans la linguistique cosérienne : "Les rapports de "signification" sont les rapports entre les signifiés des signes linguistiques [...] les rapports de "désignation" sont les rapports entre les signes linguistiques et les "objets" (la "réalité" à laquelle ils se réfèrent et qu'ils "représentent" dans le discours" (Coseriu, 2001, p. 250). D'une manière plus synthétique : «Les signes linguistiques ont des significations, au moyen desquelles ils désignent quelque chose d'extralinguistique. » (Coseriu, 2007a, p. 65, trad. CG).

23. C'est-à-dire en gardant à l'esprit tout l'enseignement critique qu'apporte la connaissance de l'histoire de l'herméneutique (les divergences d'intention théorique, les variations de statuts et de contenu du cercle herméneutique, etc.). E.g. J. Wach (1984), G. Ebeling (1959), J. Grondin (2005).

24. J'ai plaisir à remercier très chaleureusement J.-P. Durafour (Université de Tübingen) sans lequel cette traduction n'aurait pas vu le jour. Toutes les notes sont les nôtres : le genre allusif du texte rendant son contenu encore plus difficile d'accès pour un lecteur non familier d'E. Coseriu, nous sommes intervenus chaque fois que nous jugions utile d'apporter une clarification.

25. Dans ce paragraphe, «représentation ", «fonction d'expression » et "fonction d'appel » traduisent respectivement les termes allemands Darstellung, Kundgabe et Appell (employés entre guillemets par E. Coseriu) renvoyant au fameux «modèle de l'Organon » élaboré par K. Bühler (1972, 1982).

26. Le terme « référence » traduit ici Bericht, un terme introduit par F. Kainz dans sa critique du modèle de K. Bühler (Kainz, 1941-1969), qu’E. Coseriu fera sienne pour exposer sa linguistique du texte entendue comme « linguistique du sens » (Coseriu, 2007a, p. 91).

27. La conception cosérienne du sens (infra IV.2 et IV.3) découle de la distinction faite par E. Coseriu entre désignation et signification. Nous en rappelons les définitions en introduction (Interpréter le sens des textes).

28. Voir notre introduction, Pour un éclectisme raisonné.

29. Il s'agit en particulier des champs lexicaux, dont E. Coseriu (2001, p. 385-410) a proposé une importante typologie.

30. Par «système de signes" E. Coseriu entend deux choses: la «langue historique » et la «langue fonctionnelle». La langue historique est l'entité historique correspondant à une langue particulière et qu'on identifie communément au moyen d'un adjectif propre (la langue allemande, la langue française, etc.). N'étant jamais complètement homogène, la langue 
historique comprend de fait une diversité de "langues fonctionnelles», qui correspondent essentiellement aux sous-systèmes diatopique, diastratique et diaphasique. Un signe peut ainsi évoquer une identité régionale, une appartenance populaire, etc. Mais dans ce passage, l'emploi du pluriel («des systèmes») ne se justifie pas seulement par la diversité des «langues fonctionnelles " inhérentes à une même langue historique. En effet, un signe peut aussi fonctionner relativement à un autre système de signes que celui de sa langue d'appartenance : la perception d'un certain type de connotations (au sens de L.Hjelmslev) est précisément déclenchée par l'emploi d'un signe appartenant à une langue A dans le contexte d'une langue $\mathrm{B}$.

31. E. Coseriu distingue en effet, au sein de la langue, le système (Sprachsystem) et la norme ( Sprachnorm) : « la norme est un ensemble formalisé de réalisations traditionnelles ; elle comprend ce qui "existe" déjà, ce qui se trouve réalisé dans la tradition linguistique ; le système, par contre, est un ensemble de possibilités de réalisation; il comprend aussi ce qui n'a pas été réalisé, mais qui est virtuellement existant, ce qui est "possible", c'est-à-dire ce qui peut être créé selon les règles fonctionnelles de la langue" (Coseriu, 2001, p. 274). A contrario, la systématicité de la langue s'illustre dans des exceptions : en français, le participe présent du verbe savoir est sachant, mais pas savant.

32. Au sens philosophique de la " présence effective ", et par opposition à ce qui est virtuel ou en puissance.

33. Sur la notion d'écart, voir plus bas II.1.

34. «Désautomatisation » apparait en français dans les Thèses du Cercle Linguistique de Prague ( http://cercledeprague.org/documents.php). E. Coseriu tient pour malheureuse la formation de ce terme : son préfixe privatif suggère une forme de réduction, donc un concept négativement connoté, alors qu'il s'agit tout au contraire de "suppression d'une restriction ", qui est une opération positive (Coseriu, 2007a, p. 148-149).

35. En anglais dans le texte. Cette critique d'une «méthode générale de l'interprétation des textes" est développée dans E. Coseriu (2007a, p. 150-158): il ne peut en effet exister de procédure mécanique qui permette de dénombrer ou de prédire toutes les possibilités d'émergence du sens. Ce qui ne signifie pas qu'il n'existe pas de méthode adéquate pour l'interprétation des textes (voir notre introduction Interpréter le sens des textes).

36. Il faut comprendre ici « construites par l'interprétation ».

37. Il s'agit de l'approche de M. Riffaterre (1971), critiquée plus en détail dans E. Coseriu (2007a, p. 70-71), et par H. Meschonnic (1969), dans un article mémorable.

38. Référence à B. Croce (1904) qui développe ce thème dans la première partie de son Esthétique.

39. Ce passage et la formulation "pure création de significations " renvoie implicitement à Aristote et à sa définition du langage comme logos semantikos qu'E. Coseriu a maintes fois commentée (Coseriu, 2003), et qui forme la base sémiotique de la linguistique cosérienne (Hassler, 2015) : « Aristote déjà a montré que le langage en tant que tel, le "logos sémantique" (

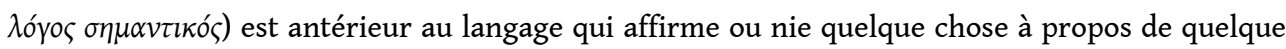
chose, au "logos propositionnel" ( non ultérieurement déterminé, on n'a pas encore, selon Aristote, la distinction entre existence et inexistence, ni la distinction entre le vrai et le faux, distinctions qui ne se présentent que dans la "proposition" (ou "jugement"), c'est-à-dire dans le logos qui transforme les rapports linguistiques en "rapports objectifs" concernant les "choses désignées". Par conséquent, le

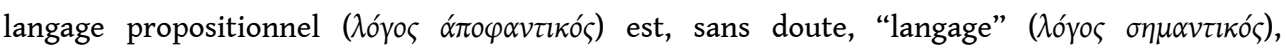
cependant non pas "langage" tout court, mais langage avec une détermination ultérieure.» (Coseriu, 2001, p. 22-23).

40. Autrement dit, « le sujet créateur de langage présuppose d'autres sujets ». Ou encore : « le moi créateur de langage suppose toujours un toi auquel il s'adresse.» (Coseriu, 2001, p. 28-29).

41. E. Coseriu fait remonter la distinction fondamentale entre "communication de quelque chose ", contingente (i.e. qui peut ne pas avoir lieu), et « communication avec un autre », nécessaire, à 
Aristote et, surtout, à G. W. F. Hegel (Phénoménologie, VI, B). Plus clairement : «Il faut, en outre, distinguer la communication pratique et contingente (la communication de quelque chose, "dire à quelqu'un ceci ou cela"), qui pourrait bien mieux se nommer "information", de la communication proprement dite, essentielle et originaire : communiquer avec quelqu'un, qui n'est pas extrinsèque au langage, puisqu'elle se manifeste même lorsque la communication pratique ne s'établit pas (c'est-à-dire, même lorsque ce qui est dit n'est pas compris). En effet, le simple "dire" est bien pour autrui, puisque le langage est, précisément, "la manifestation de soi-même pour autrui". En ce sens, parler est toujours "communiquer" [...]. » (Coseriu, 2007b, chap. III ; voir aussi Coseriu, 2001, p. 28-29).

42. En employant ces deux termes, en français dans le texte, E. Coseriu étend le périmètre traditionnel de la notion de signe (saussurien) pour concevoir le texte comme signe: « Il ne s'agit donc pas ici, en premier lieu, des relations qu'entretient le signe linguistique au sein du texte, mais de l'élaboration du "signe textuel" [Textzeichen] au moyen de la signification et de la désignation comme signifiant et du sens comme signifié. » (ibid., p. 181, trad. CG).

43. À propos de ce «niveau sémiotique supérieur" voir (Coseriu, 2007a, p. 64-67) et notre introduction (Le sens selon E. Coseriu et Interpréter le sens des textes).

\section{RÉSUMÉS}

Quelle relation la poésie entretient-elle avec le langage? Dans un texte inédit en français, dont nous produisons ici la traduction intégrale et annotée, E. Coseriu a formulé une série de thèses qui défendent l'identité entre le langage (pure activité libre ou créatrice) et la poésie (la littérature en tant qu'art). Ces thèses prennent place au sein d'une linguistique du sens, élaborée par E. Coseriu, dont nous exposons les grandes lignes dans le but de :1) discuter une définition linguistique de la poésie; 2) interroger les rapports méthodologiques entre linguistique et littérature ; 3) montrer que la linguistique cosérienne offre un cadre particulièrement adapté à l'étude linguistique des œuvres littéraires.

What is the relationship between poetry and language? Can poetry less than language? In his paper "Thesis about the theme language and poetry", that we translate here for the first time in french, E. Coseriu defends the identity of language (as purely free and creative activity) and poetry (literature as art). All these thesis take place in the "linguistic of sense" elaborated by E. Coseriu, a textual linguistic which we underline the main features in order to: 1) expose a linguistic definition of poetry; 2) discuss methodological problems concerning the linguistic study of literature; 3) demonstrate that the linguistic theory of E. Coseriu provides an efficient frame for the linguistic study of literary works.

\section{INDEX}

Mots-clés : langage, poésie, littérature, linguistique textuelle, sens, sémiotique

Keywords : language, poetry, literature, text linguistics, meaning, sense, semiotics 


\section{AUTEUR}

\section{CHRISTOPHE GÉRARD}

Université de Strasbourg, LiLPa, EA 1339, F-67000, France 